\title{
Die Revolution des Teilens - Warum, wie?
}

\author{
Jean Martin
}

Dr. med., Mitglied der Redaktion

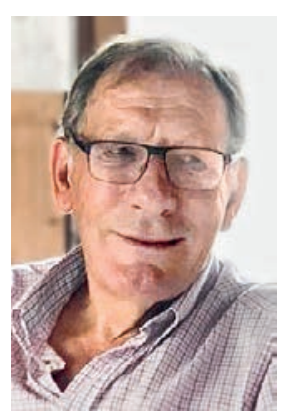

Et soudain la finance devint altruiste (Art. von J. Rambal). Le Temps (Lausanne), 30. Dezember 2017, S. 6 . La révolution philanthropique est en marche! (Interview von A.-S. Sprenger). Le Temps, 26. Mai 2018, S. 28. Siehe auch: Alexandre Mars, Le sens du partage. www.lexpress.fr/ actualite, 2. Juli 2018. La nouvelle éthique du don (Interview von L. Geffroy). Le Temps, 30. Juni 2018, S. 28. Le Temps, 20. Februar 2018, S. 10

Servigne P., Chapelle G. L'Entraide, l'autre loi de la jungle. Paris: Les Liens qui libèrent, 2017.

jean.martin[at]saez.ch
Seit Jahren schon sind die Aktionen grosser privater Geldgeber wie Bill Gates und Warren Buffett, die im Jahr 2010 die Charta "The Giving Pledge» auf den Weg brachten und Milliarden in Entwicklungsprojekte stecken (vor allem im Gesundheitsbereich, was Gates anbetrifft) eine Art Aufruf - auch für den Autor dieser Zeilen, der in den 70ern sechs Jahre in Entwicklunsprojekten tätig war und Entwicklungshilfe eher als Aufgabe von Regierungen und «nicht personalisierten» Organisationen sah. Diese Bewegung, der sich inzwischen auch Jeff Bezos (Amazon) und Mark Zuckerberg (Facebook) angeschlossen haben, steht für einen echten Paradigmenwechsel. Sie ist offensichtlich von grosser Bedeutung, und es geht nicht darum, sie abzulehnen - angesichts der Tatsache, dass sich das öffentliche Engagement rückläufig zeigt oder zumindest ins Stocken geraten ist (und auch nicht vor dem Hintergrund, dass diese Philanthropen über die Zielsetzungen der Programme entscheiden, was Fragen aufwerfen kann). In einem interessanten Artikel [1] erläutert Julie Rambal, dass die Finanzinvestoren der neuen Generation in dieselbe Richtung gehen: «Laut einer Umfrage der U.S. Trust setzen drei Viertel der Millenials bei ihren Investitionsvorhaben bevorzugt auf soziale Projekte». In der den Next Generation Leaders gewidmeten Ausgabe des Time Magazine vom 28. Mai 2018 wird der 33-jährige US Football-Star Chris Long zitiert, der sein gesamtes Grundgehalt des Jahres 2017 (1 Million USD) karitativen Zwecken zugeführt hat und zudem noch beim Einsammeln zweier weiterer Millionen aktiv beteiligt war, einfach weil er sein "gesamtes Potenzial ausschöpfen will, um die Dinge um ihn herum zu verbessern».

Private Philanthropie dieser Art ist nicht allein einer (sehr) reichen Bevölkerungsschicht vorbehalten. Für Alexandre Mars, Franzose, der sein Vermögen mit der Gründung und dem nachfolgenden Verkauf von Unternehmen gemacht hat und der sich heute als «Aktivist für das Sozialwohl» sieht, entsteht gerade eine breit gefächerte Bewegung [2]. «Es gibt eine echte Entwicklung, eine von immer mehr Menschen geteilte Sinnsuche. Immer mehr Menschen, die sich früher noch davor gescheut hatten, wollen mehr tun (...) Die vorherigen Generationen interessierten sich für sich selbst und beschränkten sich dabei auf das, was vor der eigenen Nasenspitze lag. Die Nachfolgegeneration will einen
Arbeitsplatz, der sinngebend wirkt (...) Die zweite Frage potenzieller Bewerber beim Einstellungsgespräch geht heute nicht mehr um die Grösse des Büros, sondern in welchen sozialen Projekten das Unternehmen engagiert ist» [2].

«Dies steht ganz im Zusammenhang mit dem, was wir im Alltag sehen, all die Ungleichheit, die sich nicht mehr ignorieren lässt.» Mars hat soeben ein Buch veröffentlicht (La révolution du partage, Flammarion, 2018) und ein Startup Epic gegründet.»

Der australische Philosoph Peter Singer unterrichtet Ethik in Princeton und beschäftigt sich objektiv und zweckorientiert mit der Thematik: «Effizienter Altruismus ist Philosophie und soziale Bewegung zugleich; er nutzt wissenschaftliche Erkenntnisse, um Mittel und Wege ausfindig zu machen, ein Maximum an sozialem Nutzen zu schaffen (...) Geben ist gut, doch es muss intelligent geschehen» [3]. Singer führt den Fall eines seiner Philosophie-Studierenden an, der in Oxford hätte promovieren können, es aber vorgezogen hat, in ein Finanzunternehmen an der Wall Street einzutreten, nachdem er sich ausgerechnet hatte, danach deutlich mehr an karitative Verbände abgeben zu können. Zu erwähnen die Metacharities, welche die Arbeit anderer Wohltätigkeitsorganisationen bewerten («kalte» Verfahren ... auf die Gefahr hin, dass es dabei zu technokratischen Derivaten kommen könnte? - Anmerkung J. M.).

Revolution des Teilens? Nun ... damit können sich viele als Grundprinzip einverstanden erklären, doch die Vorbehalte werden grösser, wenn unsere Eigeninteressen direkt betroffen sind - der wohlbekannte NIMBY-Effekt! («Not in my backyard» - nicht in meinem Garten). Angesichts bestimmter Diskussionen um die medizinische Vergütung stellt sich die Frage, ob eine bessere Verteilung nicht in den Rängen unserer eigenen Profession realisiert werden sollte - die Fakten machen offenkundig, dass dies alles andere als einfach ist. Bräuchten wir möglicherweise eine neue Kreativitätsethik, für die sich der junge Schweizer Jurist und Philosoph Johan Rochel stark macht [4]? Oder sollten wir uns davon überzeugen lassen, dass wir Menschen nicht von einem Wettbewerbs- und Aggressionstrieb geleitet werden, sondern die kooperativste lebende Spezies sind, wie dies Servigne et Chapelle aufzeigen [5]? 